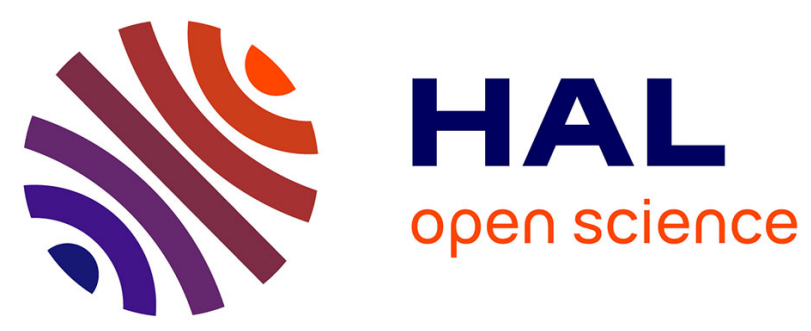

\title{
Solving an Air Conditioning System Problem in an Embodiment Design Context Using Constraint Satisfaction Techniques
}

\author{
Raphael Chenouard, Patrick Sébastian, Laurent Granvilliers
}

\section{To cite this version:}

Raphael Chenouard, Patrick Sébastian, Laurent Granvilliers. Solving an Air Conditioning System Problem in an Embodiment Design Context Using Constraint Satisfaction Techniques. Principles and Practice of Constraint Programming, Sep 2007, Providence, United States. pp.18-32, 10.1007/978-3540-74970-7_4. hal-00466675

\section{HAL Id: hal-00466675 https://hal.science/hal-00466675}

Submitted on 5 May 2010

HAL is a multi-disciplinary open access archive for the deposit and dissemination of scientific research documents, whether they are published or not. The documents may come from teaching and research institutions in France or abroad, or from public or private research centers.
L'archive ouverte pluridisciplinaire HAL, est destinée au dépôt et à la diffusion de documents scientifiques de niveau recherche, publiés ou non, émanant des établissements d'enseignement et de recherche français ou étrangers, des laboratoires publics ou privés. 


\title{
Solving an Air Conditioning System Problem using Constraint Satisfaction
}

\author{
Raphaël Chenouard ${ }^{1}$, Patrick Sébastian ${ }^{1}$, and Laurent Granvilliers ${ }^{2}$ \\ 1 ENSAM Bordeaux, TRansferts Ecoulements FLuides Energétique, CNRS, F-33405, \\ Talence Cedex \\ 2 University of Nantes, Laboratoire d'Informatique de Nantes Atlantique, CNRS, BP \\ 92208, F-44322 Nantes Cedex 3
}

\begin{abstract}
In this paper, the embodiment design of an air conditioning system (ACS) in an aircraft is investigated using interval constraint satisfaction techniques. The detailed ACS model is quite complex to solve, since it contains many coupled variables and many constraints corresponding to complex physics phenomena. Some new heuristics and notions based on embodiment design knowledge, are briefly introduced to undertake some embodiment design concepts and to obtain a more relevant and more efficient solving process than classical algorithms.

The benefits of using constraint programming in embodiment design are discussed and some difficulties for designers using CP tools are shortly detailed.
\end{abstract}

\section{An air conditioning system problem}

\subsection{Context}

The design process is a sequence of phases ranging from the definition of needs and requirements to preliminary design and detailed design (Pahl \& Beitz 1996). Preliminary design includes conceptual design leading to product schemes, and embodiment design, where feasibility studies are investigated according to geometric constraints, physics behaviors and interactions between the product, its components, and its environments.

Nowadays, no real software exist to solve complex embodiment design problems. In this context, a constraint solver, namely Constraint Explorer, was developed within a partnership between Dassault Aviation and several research laboratories. This partnership was created through the french RNTL project CO2. Our work starts from this project and aims to express some other difficulties using constraint programming in embodiment design, where problems are highly coupled according to a design point of view. We use Elisa ${ }^{3}$, an open $\mathrm{C}++$ library for constraint programming and constraint solving techniques based on interval arithmetic (Moore 1966), where we develop some new concepts and algorithms.

\footnotetext{
${ }^{3}$ http://sourceforge.net/projects/elisa/
} 
In this paper, an air conditioning system (ACS) for an aircraft (see Figure 1 ) is investigated. Figure 1 describes the main components of an ACS and functional fluxes circulation. The ACS may be viewed as a bootstrap, composed of: a turbo-machine (a compressor, a turbine and a coupling shaft), an exchanger, a trim gate, a diffuser and a nozzle. This bootstrap corresponds to a reverse Joule-Brayton cycle. A trim gate is used to regulate the air cooling system and guarantee a suitable air pressure and temperature in most of the aircraft life situations.

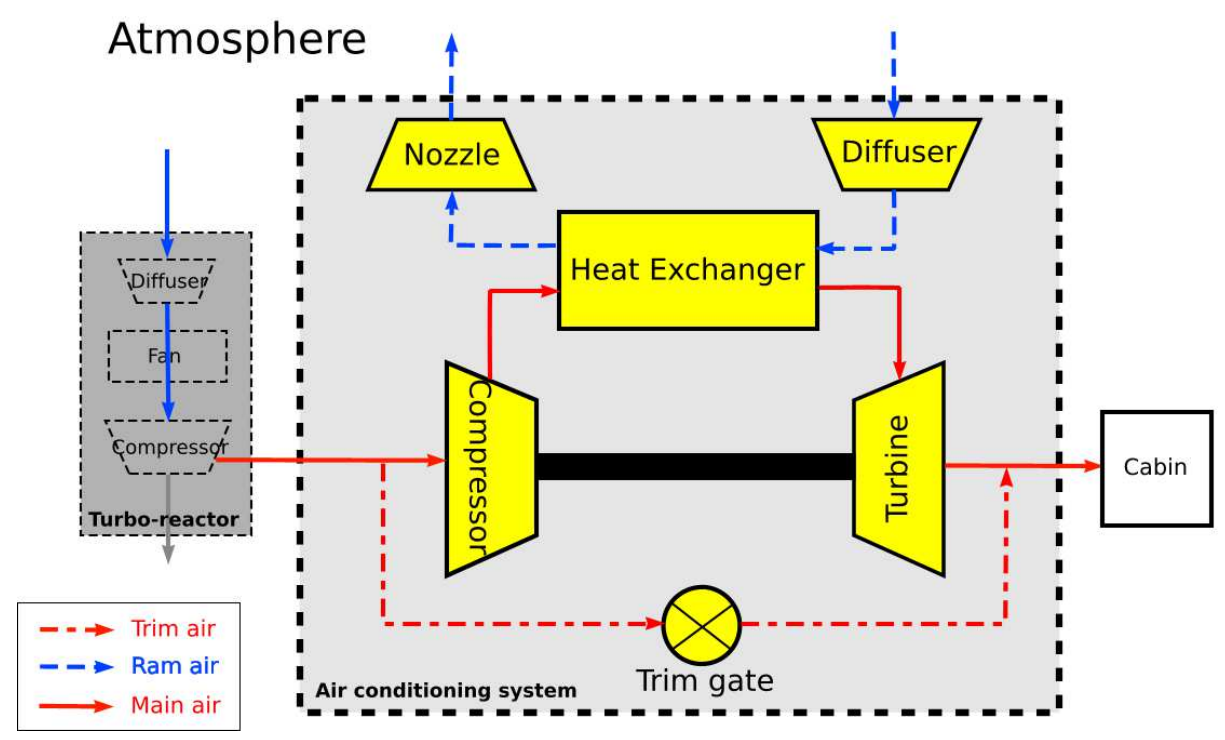

Fig. 1. Bloc functional diagram for an aircraft air conditioning system

In an aircraft, several essential criteria must be taken into account. Indeed, the air pressure and temperature of the cabin must be guaranteed for the wellbeing of the passengers, but the air flows taken from the turbo-reactor and from the atmosphere have to be carefully managed. Indeed, it can decrease the turboreactor performances and it can also increase the aircraft drag force. Moreover in an aircraft, the volume and the mass of every element penalize its performances, therefore the mass and volumes of the ACS have to be minimized.

\subsection{Model description}

In embodiment design, a product is described using several classes of variables. Indeed, designers are mainly interested in only a few variables and criteria. The design variables define by their values design solution principles. The whole design variables correspond to the smallest set of variables defining the architecture 
of a product. The auxiliary variables are used to express the constraints related to the ACS, namely physics behavior, geometric constraints and performances criteria. They are used to link design variables and criteria using some relevant knowledge related to the ACS. Criteria permit to validate and to evaluate the design solutions, but at this point, it is too early in the design process to really optimize, since some of the components may change.

All the components of the ACS are not standardized. For the sake of simplicity, only the exchanger and the trim gate are embodied, since the ACS performances seem to be mainly depending on the characteristics of the exchanger. In this way, other components are mainly expressed with efficiency coefficients (provided by manufacturers) and energy balances. Although these simplifications are used, the model of the exchanger is really complex and several coupled physics phenomena interfere.

Our model is composed of 156 variables. There are 5 design variables ( 1 for the trim gate and 4 describing the exchanger). The 151 other variables are some auxiliary variables. Most of them ( 85 variables) may be considered as explicit variables. Indeed, these variables are explicitly defined by a constraint, since they correspond to intermediary computations. They are maintained within the model, because they express well-known characteristics or criteria, which preserve the model expressivity. The 66 other auxiliary variables are mainly related to the exchanger (fins characteristics, pressures, temperatures, efficiency, etc.).

Constraints may also be classified. For instance, there are 2880 basic unary constraints describing a catalogue of 48 exchange surfaces (Kays \& London 1984). 9 constraints describe the energy balances of the 8 components used in the model. 94 other constraints express the ACS characteristics and behavior (geometry, temperature, pressure losses, etc.), most of them defining the explicit variables.

\subsection{Embodiment design solutions}

A design solution corresponds to a product architecture, which satisfy all constaints and criteria. As a consequence, a design solution is represented by design variable values. The multiple auxiliary variables values must not be taken into account when considering design solutions, since their domain exploration may induce duplicated design variable solutions. However the auxiliary variables exploration is mandatory to check the product behavior and the physics reality.

\section{Why CP?}

In Embodiment design, designers have to determine sets of design solutions to support decision making. They need tools to globally explore the solutions search space.

Stochastic methods are mainly used. They are developed in engineering departments using simulation tools coupled with optimization tools. This method 
requires the development of simulation codes, specific to the investigated problems. For instance, a tool using an optimization approach based on an evolutionary method was developed to investigate the ACS presented in this paper (Sébastian et al. 2006). These simulation codes are often powerful, but they are not easily maintainable and reusable. The solving algorithm must be redefined for each application, even though it is often time consuming to develop. In the context of a preliminary design phase, many decisions are not taken at that point and the model may evolve several times. Moreover the evolutionary approaches do not compute the entire set of solutions. In particular if some parameters of the genetic algorithm are not finely tuned, some areas of the search space may not be explored. The embodiment design phase is used to detect feasibility areas in the search space and leaving out some may be detrimental to take the appropriate decisions.

The CP approach allows to write the design models without developing their solving methods, thus the cost is lesser when a parameter or a component changes. It is already used in configuration and in conceptual design (O'Sullivan 2001, Gelle \& Faltings 2003, Yannou et al. 2003) and it seems promising for embodiment design (Zimmer \& Zablit 2001, Fischer et al. 2004). The search space is explored entirely and it is easier to determine the shapes of the solution set. This entire set of solutions allows designers to take more robust decisions, since they can investigate more easily the model response to some characteristics changes (for instance components, dimensions or criteria) and thus preserve one design architecture and some of its performances validity from future changes.

\section{How CP?}

In our air conditioning system application, we use a general CP framework based on a branch-and-prune algorithm (Van-Hentenryck et al. 1997).

\subsection{Model formulation}

We consider the classical triplet representation of CSP problems: $\langle V, D, C\rangle$. Variables are defined using discrete or continuous domains. During computations, discrete domains are converted into an interval hull and it is later refined using the discrete values it holds. The constraints are based on arithmetic expressions, using the classical equality and weak inequality operators.

However, some physics laws were established through experiments, and they are expressed as piecewise nonlinear functions. We focus on piecewise phenomena which are defined by one variable. Indeed, most physics phenomena are estimated using some representative and well-known variables (for instance in fluid mechanics: Nusselt, Reynolds, Prandtl numbers, etc.). We define a global piecewise constraint to efficiently compute theses piecewise nonlinear functions, quite similarly to Refalo's approach on linear piecewise functions (Refalo 1999). Moreover this global constraint allows to define easily the range of choices using 
a unique reference for a component, corresponding to a disjunction between all the components to choose.

\subsection{Solving algorithms}

The robust classical round-robin strategy on the variables choices for exploration often gives slow solving times for embodiment design problems and generates many duplicated design solutions. The main explanation is the useless computation steps made on auxiliary variables. That's why we develop a heuristic custom search based on variable classes linked to the embodiment design point of view. Indeed, design variables have defined the main structuring characteristics and models are often established around them and around criteria. Auxiliary variables are only introduced to express performance criteria, physics behavior, etc. (see figure 2).

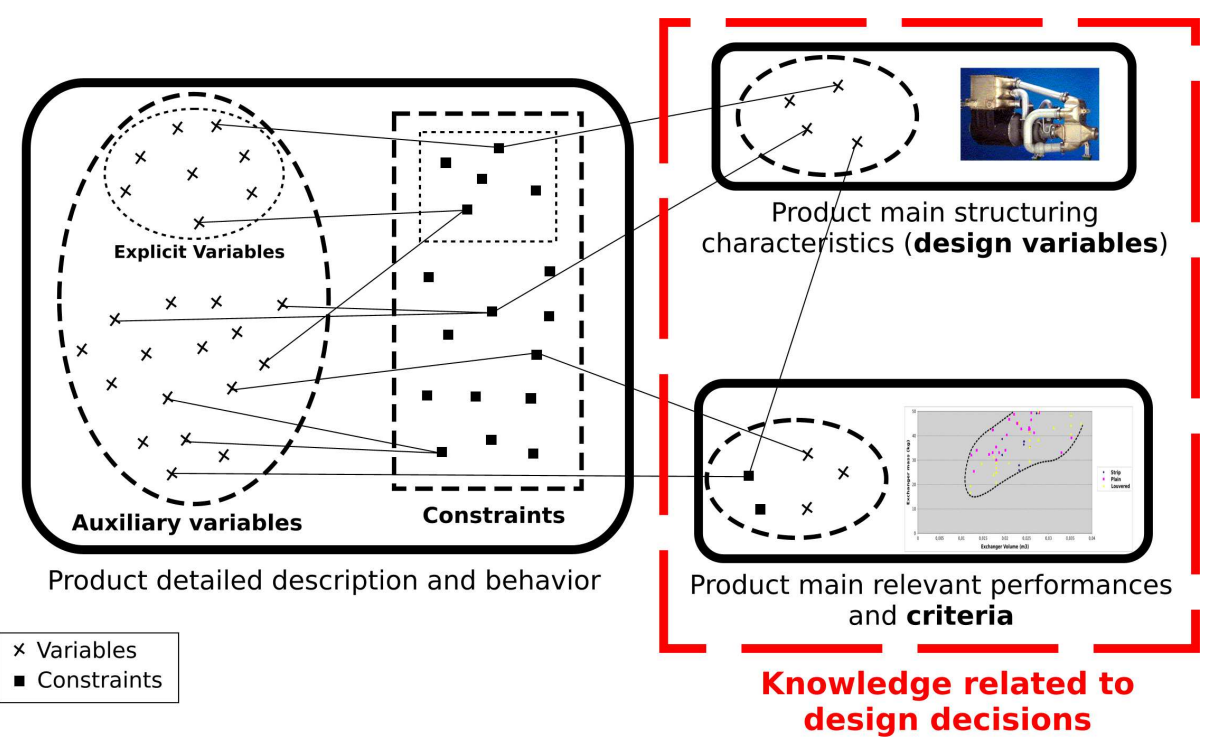

Fig. 2. Knowledge classification in an embodiment design model

In addition, some of the auxiliary variables are explicitly defined according to other variables. They correspond to intermediary computation steps, but they are maintained in the model to ensure its intelligibility. To avoid some useless exploration steps according to these variables, their precision are defined to the infinite value, so that their precisions are always achieved. Their values are computed by the consistency pruning process based on $2 \mathrm{~B}$ (Lhomme 1993) and box consistency (Benhamou et al. 1999). It is important to highlight, that explicit variables may be defined using other explicit variables. In this case, no 
dependency cycle has to stand between them. Some small design problems are presented using such variables or not in Vu's work (Vu 2005).

These algorithms and the use of explicit variables allow us to compute more efficiently the entire solution set of embodiment design problems in a few hours, whereas other algorithms do not compute the first solution after several hours.

In some other works, models are decomposed in small blocks organized in a directed acyclic graph (Reddy et al. 1996, Bliek et al. 1998, Neveu et al. 2006). It may be interpreted to a variables solving order, where blocks are fully solved before starting the next one in the graph. It is an efficient approach for problems, where coupled variables are not too numerous. Indeed, when there are several sets of coupled variables, the model is decomposed in big blocks and the hardness of the problem remains. Moreover, preliminary design problems are often under-constrained, and many start variables have to be chosen to compute a decomposition of the constraint network. In this case, these variable domains are explored almost exhaustively and it may induce useless computation steps on irrelevant variables.

\subsection{Precision management}

The management of the precisions variables appears to be fundamental to ensure a relevant solution set for designers. Indeed, each design variable has a precision representing the interval width, which defines solutions according to the design point of view. These precisions are linked with tolerances and real-world feasibility.

In many cases, a global precision for all variables (for instance $10^{-8}$ is the default precision on several solvers) has no sense considering the design point of view. A default precision can be defined for some variables, but most relevant variables of an embodiment design problem must have their own appropriate precision. The design variable precisions have to be defined taking into account the product specifications and requirements.

The precisions on auxiliary variables are more difficult to set properly. Some complex phenomena may be taken into account and designers may have difficulties to forecast the relevant variable precisions linked with these phenomena. Indeed, they may use some confidence percentage linked to some imprecisions related to the computed values. These imprecisions are linked to simplifying assumptions, which allow designers to neglect terms when writing balance equations. From this point of view, balance equations of mass, energy or momentum are linked to a precision taking into account the terms neglected by designers. This confidence percentage may be associated as a relative precision to some relevant variables of constraints, for instance efficiency coefficients. However some other auxiliary variables may be related to some physics quantities or to some physics order of magnitude and their precisions (absolute or relative) should be defined taking this knowledge into account.

The precision management is really fundamental to obtain efficient solving time (not completely useless search space explorations) and relevant solutions (not too many duplicated design solutions). 


\subsection{Model implementation}

All the constants, variables, domains and constraints of the ACS problem are detailed in the following tables. The $c$ or $h$ indexes related to some variables identify the cold or hot side of the exchanger. We do not define the domain of the variables from the Kays \& London database, since it is useless.

\begin{tabular}{|l|c|}
\hline Constants & \\
\hline \hline Air properties & $C p=1000 \mathrm{~J} / \mathrm{kg} . \mathrm{K}, r=287 \mathrm{~J} / \mathrm{kg} / \mathrm{K}, \gamma=1.4$ \\
Flight conditions & $Z=10500 \mathrm{~m}, M=0.8$ \\
Turbo-reactor characteristics & $T C_{T R}=8, \eta_{T R d}=0.9, \eta_{T R}=0.8$ \\
Components efficiency & $\eta_{c}=0.75, \eta_{A T}=0.95, \eta_{t}=0.8, \eta_{N O}=0.9, \eta_{D I}=0.9$ \\
Air sent into the cabin & $T_{5}=278.15 \mathrm{~K}, p_{5}=85000 \mathrm{~Pa}, q=0.7 \mathrm{~kg} / \mathrm{s}$ \\
Plates characteristics & $k p=20 \mathrm{~W} / \mathrm{m} / \mathrm{K}, t p=0.001 \mathrm{~m}$ \\
\hline
\end{tabular}

\begin{tabular}{|l|c|}
\hline Design variables & \\
\hline Width of the exchanger $(\mathrm{m})$ & $\mathrm{Lx} \in[0.1,1], \mathrm{p}(\mathrm{Lx})=0.01$ \\
Mass capacity ratio $(-)$ & $\tau \in[1,8], \mathrm{p}(\tau)=0.5$ \\
Hot-side exchange surface reference $(-)$ & ExSur $f_{h} \in[1,48]:$ int \\
Cold-side exchange surface reference $(-)$ & ExSur $f_{c} \in[1,48]:$ int \\
Trim gate radius $(\mathrm{m})$ & $\operatorname{Rv} \in\{0.01,0.02, \ldots, 0.2\}$ \\
\hline
\end{tabular}

\begin{tabular}{|l|c|}
\hline Auxiliary variables & \\
\hline \hline Exchanger material characteristics & roex $\in[0,10000], k w \in[0,500]$ \\
\hline Exchanger pressures $(\mathrm{Pa})$ & $p_{2} \in[0,1000000] \wedge p\left(p_{2}\right)=5 \%$, \\
& $p_{3} \in[0,1000000] \wedge p\left(p_{3}\right)=5 \%$ \\
& $p e_{i c} \in[0,1000000] \wedge p\left(p e_{o c}\right)=5 \%$, \\
& $p e_{o c} \in[0,1000000] \wedge p\left(p e_{o c}\right)=5 \%$ \\
\hline Exchanger temperatures (K) & $T_{2} \in[0,1000] \wedge p\left(T_{2}\right)=5 \%$, \\
& $T_{3} \in[0,1000] \wedge p\left(T_{3}\right)=5 \%$ \\
& $T e_{i c} \in[0,1000] \wedge p\left(T e_{i c}\right)=5 \%$, \\
& $T e_{o c} \in[0,1000] \wedge p\left(T e_{o c}\right)=5 \%$ \\
\hline Exchanger pressure losses $(\mathrm{Pa})$ & $\Delta P e_{h} \in[-\infty,+\infty] \wedge p\left(\Delta P e_{h}\right)=10 \%$ \\
& $\Delta P e_{c} \in[-\infty,+\infty] \wedge p\left(\Delta P e_{c}\right)=10 \%$ \\
\hline Exchanger efficiency coefficient (-) & $\epsilon \in[0,1] \wedge p(\epsilon)=5 \%$ \\
\hline Air flows in the exchanger $(\mathrm{kg} / \mathrm{s})$ & $q_{r a} \in[0,+\infty] \wedge p\left(q_{r a}\right)=0.001$ \\
& $q_{m a} \in[0,+\infty] \wedge p\left(q_{m a}\right)=0.001$ \\
\hline Pressure after the turbine $(\mathrm{Pa})$ & $p_{4} \in[0,1000000] \wedge p\left(p_{4}\right)=5 \%$ \\
\hline Temperature after the turbine $(\mathrm{K})$ & $T_{4} \in[0,1000] \wedge p\left(T_{4}\right)=5 \%$ \\
\hline
\end{tabular}

\begin{tabular}{|l|c|}
\hline $\begin{array}{l}\text { Auxiliary variables } \\
\text { relating to the K\&L database }\end{array}$ & \\
\hline \hline Fins characteristics & $b_{h}, r h_{h}, \delta_{h}, \beta_{h}, b_{c}, r h_{c}, \delta_{c}, \beta_{c}$ \\
\hline Colburn interpolation coefficients & $J S_{h 1}, J S_{h 2}, J S_{h 3}, J S_{c 1}, J S_{c 2}, J S_{c 3}$ \\
\hline
\end{tabular}




\begin{tabular}{|l|c|}
\hline Fanning interpolation coefficients & $f S_{h 1}, f S_{h 2}, f S_{h 3}, f S_{c 1}, f S_{c 2}, f S_{c 3}$ \\
\hline Kc interpolation coefficients & $K c S_{h 1}, K c S_{h 2}, K c S_{h 3}, K c S_{h 4}, K c S_{h 5}, K c S_{h 6}$ \\
& $K c S_{h 7}, K c S_{h 8}, K c S_{h 9}$ \\
& $\left.K c S_{c 1}, K c S_{c 2}, K c S_{c 3}, K c S_{c 4}, K c S_{c 5}, K c S_{c 6}\right]$ \\
\hline Ke interpolation coefficients & $K c S_{c 7}, K c S_{c 8}, K c S_{c 9}$ \\
\hline & $K e S_{h 1}, K e S_{h 2}, K e S_{h 3}, K e S_{h 4}, K e S_{h 5}, K e S_{h 6}$ \\
& $K e S_{h 7}, K e S_{h 8}, K e S_{h 9}$ \\
& $K e S_{c 1}, K e S_{c 2}, K e S_{c 3}, K e S_{c 4}, K e S_{c 5}, K e S_{c 6}$ \\
& $K e S_{c 7}, K e S_{c 8}, K e S_{c 9}$ \\
\hline
\end{tabular}

\begin{tabular}{|c|c|}
\hline $\begin{array}{l}\text { Auxiliary variables } \\
\text { defined as explicit variables }\end{array}$ & \\
\hline Exchanger dimensions $(\mathrm{m})$ & $L y=L x, L z=0.25 * L x$ \\
\hline Fins characteristics & $\begin{array}{c}\alpha_{h}=\frac{\left(b_{h} * \beta_{h}\right)}{\left(b_{h}+b_{c}+2 * \delta_{h}\right)}, \alpha_{c}=\frac{\left(b_{c} * \beta_{c}\right)}{\left(b_{h}+b_{c}+2 * \delta_{c}\right)} \\
\sigma_{h}=\alpha_{h} * r h_{h}, \sigma_{c}=\alpha_{c} * r h_{c}\end{array}$ \\
\hline Fins efficiency & $\eta f_{h}=\frac{\tanh m l_{h}}{m l_{h}}, \eta f_{c}=\frac{\tanh m l_{c}}{m l_{c}}$ \\
\hline Exchange surface dimensions & $\begin{array}{c}A_{h}=\alpha_{h} *(L x * L y * L z), A_{c}=\alpha_{c} *(L x * L y * L z) \\
A w=L x * L y * n, A c_{h}=\sigma_{h} * A f_{h}, A c_{c}=\sigma_{c} * A f_{c} \\
A f_{h}=L x * L z, A f_{c}=L y * L z\end{array}$ \\
\hline Exchange surface efficiency & $\eta 0_{h}=1-\frac{A f_{h}}{A_{h}} *\left(1-\eta f_{h}\right), \eta 0_{c}=1-\frac{A f_{c}}{A_{c}} *\left(1-\eta f_{c}\right)$ \\
\hline Exchanger inner wall thickness $(\mathrm{m})$ & $t w=\frac{\left(\delta_{h}+\delta_{c}\right)}{2}$ \\
\hline Number of plates $(-)$ & $n=\frac{L z}{b_{h}+b_{c}+2 * \delta_{h}}$ \\
\hline Air characteristics in the exchanger & $\begin{array}{c}G_{h}=\frac{q m a}{A c_{h}}, G_{c}=\frac{q_{r a}}{A c_{c}}, \\
\mu_{h}=\frac{-1.075 e^{-5}-2.225 e^{-9} * T_{3}+1.725 e^{-6} * \sqrt{T_{3}}}{2}+ \\
\frac{-1.075 e^{-5}-2.225 e^{-9} * T_{2}+1.725 e^{-6} * \sqrt{T_{2}}}{2} \\
\mu_{c}=\frac{-1.075 e^{-5}-2.225 e^{-9} * T e_{o c}+1.725 e^{-6} * \sqrt{T e_{o c}}}{2}+ \\
\frac{-1.075 e^{-5}-2.225 e^{-9} * T e_{i c}+1.725 e^{-6} * \sqrt{T e_{i c}}}{2} \\
\rho_{2}=\frac{p_{2}}{r * T_{2}}, \rho_{3}=\frac{p_{3}}{r * T_{3}}, \\
\rho_{i c}=\frac{p e_{i c}}{r * T e_{i c}}, \rho_{o c}=\frac{p e_{o c}}{r * T e_{o c}}, \\
C p_{o h}=1003.7+6.8 e^{-2} *\left(T_{3}-273.15\right)+ \\
2.22 e^{-4} *\left(T_{3}-273.15\right)^{2} \\
C p_{i h}=1003.7+6.8 e^{-2} *\left(T_{2}-273.15\right)+ \\
2.22 e^{-4} *\left(T_{2}-273.15\right)^{2} \\
C p_{h}=\frac{C p_{o h}+C p_{i h}}{2} \\
C p_{o c}=1003.7+6.8 e^{-2} *\left(T e_{o c}-273.15\right)+ \\
2.22 e^{-4} *\left(T e_{o c}-273.15\right)^{2} \\
C p_{i c}=1003.7+6.8 e^{-2} *\left(T e_{i c}-273.15\right)+ \\
2.22 e^{-4} *\left(T e_{i c}-273.15\right)^{2} \\
C p c=\frac{C p_{o c}+C p_{i c}}{2} \\
\end{array}$ \\
\hline Exchanger mass (kg) & $m_{e x}=\rho_{e x} * L x * L y *\left(\left(\frac{\sigma_{h} * \delta_{h}}{r h_{h}}+\frac{\sigma_{c} * \delta_{c}}{r h_{c}}\right) * L z+n * t w\right)$ \\
\hline Exchanger volume (m3) & $V=L x * L y * L z$ \\
\hline Air speed in the exchanger $(\mathrm{m} / \mathrm{s})$ & $C_{h}=\frac{q_{m a}}{A c_{h} * \rho_{2}}, C c=\frac{q_{r a}}{A c_{c} * \rho_{i c}}$ \\
\hline Heat transfer characteristics & $N u t=\frac{\lambda=\frac{q_{r a} * C p_{i c}}{q_{m a} * C p_{i h}},}{\frac{q_{m a} * C p_{i h}}{\eta 0_{h} * h_{h} * A_{h}}+\frac{t}{k w * A w}+\frac{1}{\eta 0_{c} * h_{c} * A_{c}}}$, \\
\hline
\end{tabular}




\begin{tabular}{|c|c|}
\hline & $\begin{array}{c}h_{h}=J_{h} * G_{h} * C p_{h} * P r_{h}^{-2 / 3} \\
h c=J_{c} * G_{c} * C p_{c} * P r_{c}^{-2 / 3}\end{array}$ \\
\hline Colburn Coefficients (-) & $\begin{array}{c}J_{h}=e^{J S_{h 1} * \log ^{2}\left(R e_{h}\right)+J S_{h 2} * \log \left(R e_{h}\right)+J S_{h 3}}, \\
J_{c}=e^{J S_{c 1} * \log ^{2}\left(R e_{c}\right)+J S_{h 2} * \log \left(R e_{c}\right)+J S_{h 3}}\end{array}$ \\
\hline Fanning friction factor $(-)$ & $\begin{array}{c}f_{h}=e^{f S_{h 1} * \log ^{2}\left(R e_{h}\right)+f S_{h 2} * \log \left(R e_{h}\right)+f S_{h 3}}, \\
f_{c}=e^{f S_{c 1} * \log ^{2}\left(R e_{c}\right)+f S_{h 2} * \log \left(R e_{c}\right)+f S_{h 3}}\end{array}$ \\
\hline Prandtl number (-) & $\begin{array}{c}\operatorname{Pr}_{h}=\frac{\left(0.825-0.00054 * T_{3}+5.0 e-7 * T_{3}^{2}\right)}{2}+ \\
\frac{\left(0.825-0.00054 * T_{2}+5.0 e^{-7} * T_{2}^{2}\right)}{2} \\
\operatorname{Pr}_{c}=\frac{0.825-0.00054 * T e_{o c}+5.0 e^{-7} * T e_{o c}^{2}}{2}+ \\
\frac{0.825-0.00054 * T e_{i c}+5.0 e^{-7} * T e_{i c}^{2}}{2}\end{array}$ \\
\hline Reynolds number (-) & $R e_{h}=\frac{4 * r h_{h} * G_{h}}{\mu_{h}}, R e_{c}=\frac{4 * r h_{c} * G_{c}}{\mu_{c}}$ \\
\hline Inlet pressure losses coefficient & $\begin{array}{c}K c_{h}=K c S_{h 1} * \sigma_{h}^{2}+K c S_{h 2} * \sigma_{h}+K c S_{h 3} * \log ^{2}\left(R e_{h}\right)+ \\
K c S_{h 4} * \sigma_{h}^{2}+K c S_{h 5} * \sigma_{h}+K c S_{h 6} * \log \left(R e_{h}\right)+ \\
K c S_{h 7} * \sigma_{h}^{2}+K c S_{h 8} * \sigma_{h}+K c S_{h 9} \\
K c_{c}=K c S_{c 1} * \sigma_{c}^{2}+K c S_{c 2} * \sigma_{c}+K c S_{c 3} * \log ^{2}\left(R e_{c}\right)+ \\
K c S_{c 4} * \sigma_{c}^{2}+K c S_{c 5} * \sigma_{c}+K c S_{c 6} * \log \left(R e_{c}\right)+ \\
K c S_{c 7} * \sigma_{c}^{2}+K c S_{c 8} * \sigma_{c}+K c S_{c 9}\end{array}$ \\
\hline Outlet pressure losses coefficient & $\begin{array}{c}K e_{h}=K e S_{h 1} * \sigma_{h}^{2}+K e S_{h 2} * \sigma_{h}+K e S_{h 3} * \log ^{2}\left(R e_{h}\right)+ \\
K e S_{h 4} * \sigma_{h}^{2}+K e S_{h 5} * \sigma_{h}+K e S_{h 6} * \log \left(R e_{h}\right)+ \\
K e S_{h 7} * \sigma_{h}^{2}+K e S_{h 8} * \sigma_{h}+K e S_{h 9} \\
K e_{c}=K e S_{c 1} * \sigma_{c}^{2}+K e S_{c 2} * \sigma_{c}+K e S_{c 3} * \log ^{2}\left(R e_{c}\right)+ \\
K e S_{c 4} * \sigma_{c}^{2}+K e S_{c 5} * \sigma_{c}+K e S_{c 6} * \log \left(R e_{c}\right)+ \\
K e S_{c 7} * \sigma_{c}^{2}+K e S_{c 8} * \sigma_{c}+K e S_{c 9}\end{array}$ \\
\hline Atmosphere characteristics & $T_{a}=288.2-0.00649 * Z, p_{a}=101290 *\left(\frac{T_{a}}{288.08}\right)^{5.256}$ \\
\hline Turbo-reactor temperatures (K) & $\begin{array}{c}T_{0}=T_{a} *\left(1+\frac{M^{2} *(\gamma-1)}{2}\right) \\
T_{1}=T_{0} *\left(1+\frac{1}{\eta_{T R c}} *\left(\left(\frac{p 1}{p 0}\right)^{\frac{\gamma-1}{\gamma}}-1\right)\right)\end{array}$ \\
\hline Turbo-reactor pressures $(\mathrm{Pa})$ & $\begin{array}{c}p_{0}=p_{a} *\left(\eta_{T R d} * \frac{M^{2} *(\gamma-1)}{2}+1\right)^{\frac{\gamma}{\gamma-1}} \\
p_{1}=T C_{T R} * p_{0}\end{array}$ \\
\hline $\begin{array}{l}\text { Diffuser pressures }(\mathrm{Pa}) \\
\text { and temperatures }(\mathrm{K})\end{array}$ & $\begin{array}{c}p o_{D c}=p i_{D c} *\left(\eta_{D I} * M^{2} * \frac{\gamma-1}{2}+1\right)^{\frac{\gamma}{\gamma-1}} \\
T o_{D c}=T i_{D c} *\left(1+M^{2} * \frac{\gamma-1}{2}\right) \\
p i_{D c}=p_{a}, T i_{D c}=T_{a}\end{array}$ \\
\hline $\begin{array}{l}\text { Nozzle pressure }(\mathrm{Pa}) \\
\text { and temperatures }(\mathrm{K})\end{array}$ & $\begin{array}{c}p i_{B c}=p e_{o c}, p o_{B c}=p_{a}, T i_{B c}=T e_{o c} \\
T o_{B c}=T i_{B c} *\left(1+\eta_{N O} *\left(-1+\left(\frac{p o_{B c}}{p i_{B c}}\right)^{\frac{\gamma-1}{\gamma}}\right)\right)\end{array}$ \\
\hline Trim gate descripion & $\begin{array}{c}\nu=\frac{r * T_{1}}{p_{1}}, p_{4}=p_{5}, q_{m a v}=q-q_{m a} \\
k s i_{\alpha}=e^{\left(4.03108 e^{-4} * \alpha^{2}+8.50089 e^{-2} * \alpha-1.59295\right.}\end{array}$ \\
\hline Various explicit variables & $\begin{array}{c}m_{h}=\sqrt{\frac{2 * h_{h}}{k w * \delta_{h}}}, m_{c}=\sqrt{\frac{2 * h_{c}}{k w * \delta_{c}}}, \\
m l_{h}=\frac{m_{h} * b_{h}}{2}, m l_{c}=\frac{m_{c} * b_{c}}{2}, \\
\operatorname{CPam}_{h}=K c_{h}+1-\left(\sigma_{h}^{2}\right), C P d_{h}=2 *\left(\frac{\rho_{2}}{\rho_{3}}-1\right), \\
C P r_{h}=f_{h} * \frac{A_{h}}{A c_{h}} *\left(2 * \frac{\rho_{2}}{\rho_{2}+\rho_{3}}\right), \\
C \operatorname{Pav}_{h}=\left(K e_{h}+\sigma_{h}^{2}-1\right) * \frac{r h o 2}{r h o 3}, \\
C \operatorname{Pam}_{c}=K c_{c}+1-\sigma_{c}^{2}, C P d_{c}=2 *\left(\frac{\rho_{i c}}{\rho_{o c}}-1\right), \\
C P r_{c}=f_{c} * \frac{A_{c}}{A c_{c}} *\left(2 * \frac{\rho_{i c}}{\rho_{i c}+\rho_{o c}}\right), \\
C \operatorname{Pav}_{c}=\left(K e_{c}+\sigma_{c}^{2}-1\right) * \frac{\rho_{i c}}{\rho_{o c}}\end{array}$ \\
\hline
\end{tabular}




\begin{tabular}{|c|c|}
\hline Constraints & \\
\hline $\begin{array}{l}\text { Catalogue of } \\
\text { exchange surfaces }\end{array}$ & $\begin{array}{l}\operatorname{exchSurf}_{h}=1 \rightarrow J S_{h 1}=0.0314 \wedge \cdots \wedge K c S_{h 9}=1.717231 \\
\vdots \\
\operatorname{exchSurf}_{h}=48 \rightarrow J S_{h 1}=0.0369 \wedge \cdots \wedge K c S_{h 9}=1.551097 \\
\operatorname{exchSurf}_{c}=1 \rightarrow J S_{c 1}=0.0314 \wedge \cdots \wedge K c S_{c 9}=1.717231 \\
\vdots \\
\text { exchSurf }_{c}=48 \rightarrow J S_{c 1}=0.0369 \wedge \cdots \wedge K c S_{c 9}=1.551097\end{array}$ \\
\hline $\begin{array}{l}\text { Materials choice for the } \\
\text { exchanger }\end{array}$ & $\begin{array}{l}T_{2} \leq 473 \rightarrow \text { roe } x=8440 \wedge k w=30 \\
T_{2} \geq 473 \rightarrow \text { roe } x=8440 \wedge k w=30\end{array}$ \\
\hline $\begin{array}{l}\text { Exchanger description: } \\
\text { Air flow ratio } \\
\text { Heat efficiency } \\
\text { for the hot-side } \\
\text { Hot-side energy balance } \\
\text { Hot-side pressure losses } \\
\text { Cold-side energy balance } \\
\text { Cold-side pressure losses }\end{array}$ & 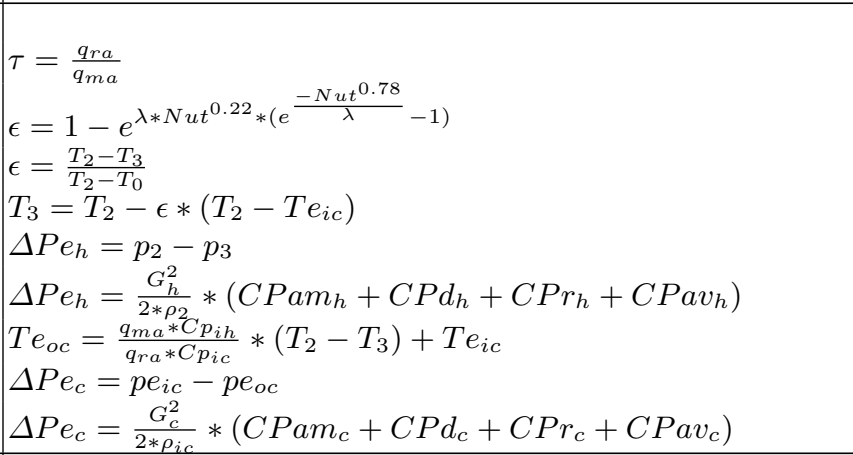 \\
\hline $\begin{array}{l}\text { Components energy } \\
\text { balances: } \\
\text { Compressor } \\
\text { Coupling shaft } \\
\text { Turbine } \\
\text { Trim gate }\end{array}$ & $\begin{array}{l}\eta_{c} *\left(\frac{T_{2}}{T_{1}}-1\right)=\left(\frac{p_{2}}{p_{1}}\right)^{\frac{\gamma}{\gamma-1}}-1 \\
\left(T_{2}-T_{1}\right)=\eta_{A T} *\left(T_{3}-T_{4}\right) \\
1-\mathrm{T} 4 / \mathrm{T} 3=\eta_{t} *\left(1-\left(\frac{p_{4}}{p_{3}}\right)^{\frac{\gamma-1}{g a m m a}}\right) \\
q * C p * T_{5}=q_{m a} * C p * T_{4}+q_{m a v} * C p * T_{1}\end{array}$ \\
\hline & $\begin{array}{l}p_{1}-p_{4}=k s i_{\alpha} * \nu * \frac{q_{m a v}^{2}}{2 *\left(\pi * R v^{2}\right)^{2}} \\
T o_{D c}=T e_{i c} \\
p o_{D c}=p e_{i c}\end{array}$ \\
\hline
\end{tabular}

\subsection{ACS solutions}

Using all this knowledge about the ACS model during the solving process permit us to compute a finite set of 322 design solution principles in a few hours on a personal computer at $2 \mathrm{GHz}$ (see figure 3 and 4). Using a classical Round-Robin strategy with relevant precisions, but without taking into account design variable classes, give a set of 388608 solutions in several hours.

Figure 3 illustrates the distribution of the solution set considering 2 major criteria: the volume of the exchanger and its mass. The three symbols used to plot solutions represent the exchange surface types used for the hot-side in the exchanger. Figure 4 shows the solutions sorted by the exchange surface references from the catalogue for the hot side of the exchanger. It is important to highlight that there are no solutions with the wavy exchange surfaces from the catalogue and the best ones are from the louvered exchange surface family. 


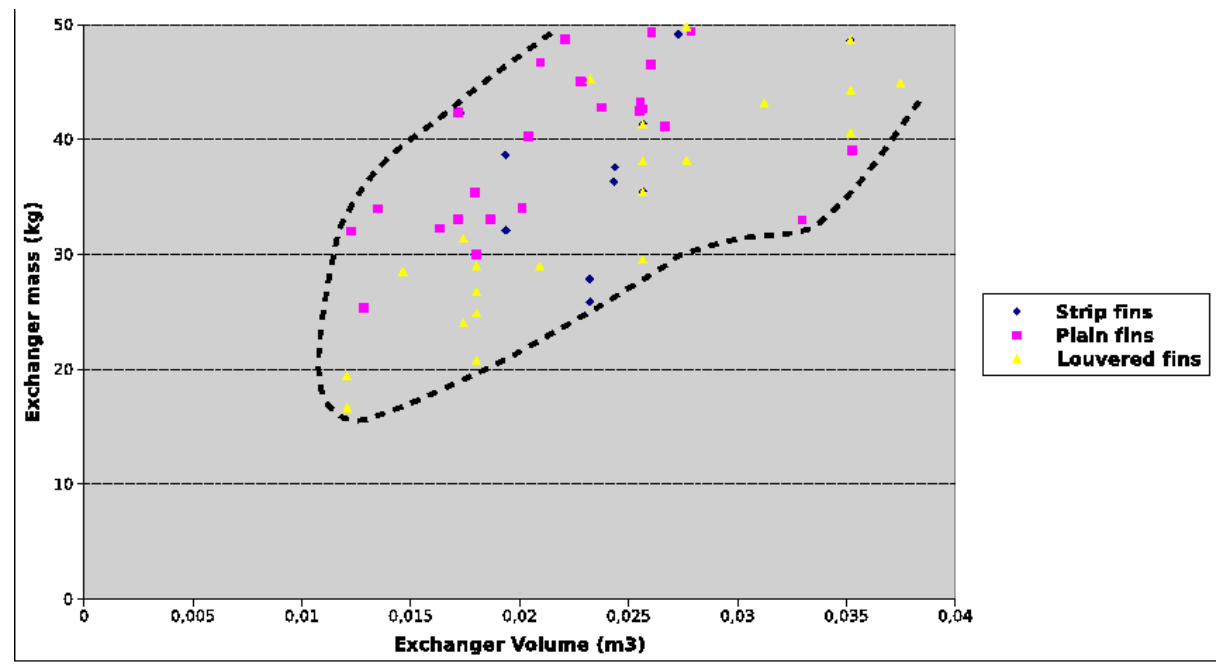

Fig. 3. ACS solutions considering the main system performance criteria: volume and mass of the exchanger.

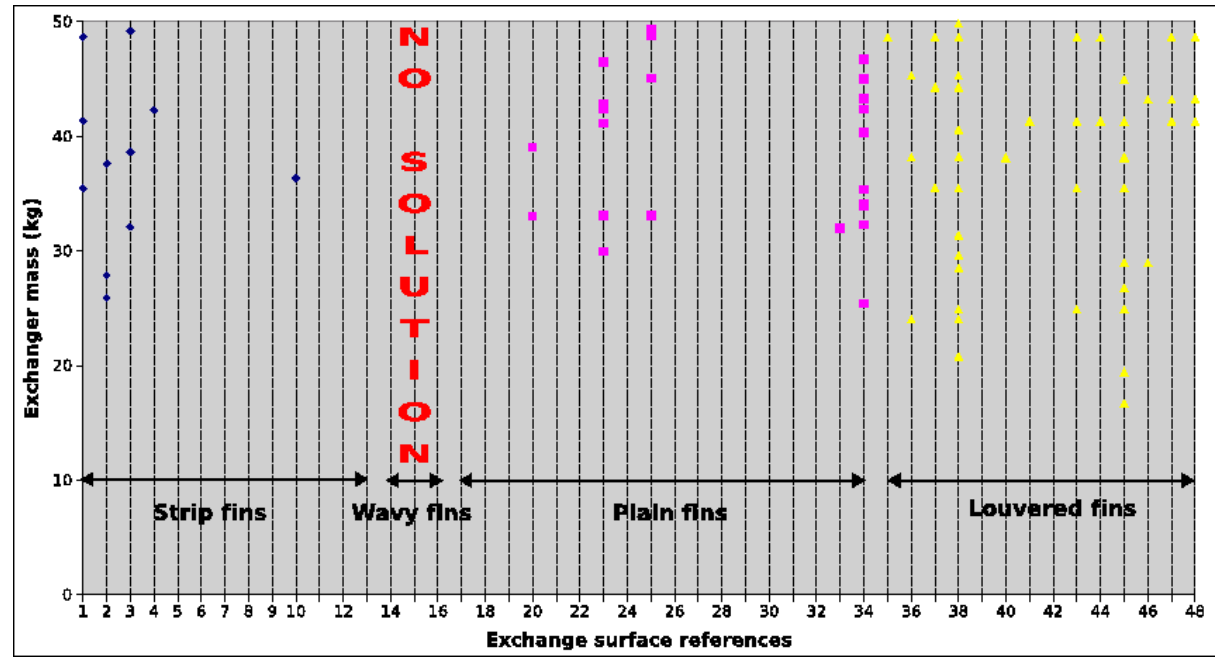

Fig. 4. ACS solutions considering the exchanger mass sorted by hot-side exchange surface references. 
Considering all this results, designers may have a better understanding of the solution set of the ACS. These results allow us to validate some factual knowledge about the design of air conditioning systems in an aircraft. Some exchange surfaces families appear to participate in more powerful ACS and itcould save time in the upstream phases of ACS design.

\section{Added value of CP?}

\subsection{Development cost}

This application was developed in several steps, because of the complexity of the model. A first model without fins between the exchanger plates was developed. It took a few days to get a preliminary model, since we used the data from an evolutionary approach and previous work on this system. However several days were needed to get a model with valid physics behaviors. Finally the entire model with fins data was built after several weeks. We do not count the development time of the heuristics search in the solver.

However for new projects, we suppose that the model writing phase take longer if it is as complex as for the ACS, but we think that only a few weeks could be necessary to devise a CSP model for design problems, particularly if it is preceded by a relevant analysis of the product. It permits to identify and organise into a hierarchy the components and physics phenomena (Szykman et al. 1999, Scaravetti 2004). Then the main characteristics of the product are defined and only the relevant physics laws and the relevant performances criteria are expressed.

\subsection{Industrial use}

Our work on the ACS is based on previous work using other solving methods. The CP approach allows to avoid the test and fail method commonly used in design process. In the classical approach, a model is developed and then criteria validate or not the solutions found.In the CP approach, criteria are within the model and only satisfactory solutions are computed. In addition, each model development (embodiment design and detailed design) takes about 3 months and the deadlines of industrial design are incompatible with several model developments.

Our ACS model is quite complete considering an embodiment stage, since it takes into account more detailed knowledge related to components and their performances than previously used models, namely the exchanger and its exchange surfaces. The results from an equivalent model were taken into account for the design of the ACS in a vehicle subjected to confidentiality constraints developed by Dassault Aviation.

\subsection{Feedback and user experience}

The use of constraint satisfaction techniques in embodiment design appears promising, although the solving process is not always efficient. Indeed, solving 
some models may be time consuming compared to the evolutionary approach and the solution set is not always relevant, since there are many duplicated solutions realted to the design point of view.

We think that most CP tools or constraints solvers are not enough designed for beginners in constraints and particularly designers, although the constraints languages are often intuitive. Solvers are mainly concerned with mathematical results, leaving aside other needs. Most solvers do not take into account fundamental notions of design, as for instance the design and auxiliary variables, explicit variables, piecewise constraints, matrix calculus, models and data from catalogues or databases, etc. More to the point, the results obtained with a constraint solver are not reusable for the next phases of the design process, where CAD tools are mainly used.

Moreover, the development of real applications with many constraints and large amounts of data is difficult. Indeed, the inconsistencies are often complex to explain, since only little information is available about the solving failures and only how familiar the user is with the problem may really help. In our application, this lack of information about the solving process and the inconsistencies have drastically increased the development time of the ACS model.

Finally, we think that solvers must not be some black box tools. They have to be flexible and tuneable to fit every problem type. The definition of heuristics at several levels should make it possible to undertake all model specificities, like, for instance, considering the relevance of variable classes according to a design point of view, or more specific strategies related to the investigated problem.

\section{Conclusion}

In this paper, the model of an air conditioning system in an aircraft is investigated according to the embodiment design context. Indeed, some simplifications are made on several components, whereas others are more detailed, adding the reality of complex physics phenomena at several scales. Some new concepts and new heuristics are defined according to variable classes and relevant precisions in order to support decision making. Indeed, many duplicated design solutions are eliminated when compared to a classical CP solving approach and the entire set of design solutions is computed. These new notions increase the efficiency of the solving process, but we believe that some improvements can still be made to undertake additional design model specificities.

Solving embodiment design models with constraint programming seems promising, although some difficulties may appear for beginners in constraints. Indeed, most solvers are mathematical tools, where design concepts are missing. The difficulty for CP is to keep within a general approach to solve problems in order to keep its easy-to-use feature, and at the same time to solve efficiently all types of problems.

However, several fundamental notions may be integrated in solvers to help designers with their task, as for instance the variable types in design, piecewise constraints, etc. The constraints solvers should also be linked with other tools 
commonly used in design (CAD tools), and perhaps use an object or component oriented approach to express constraints and to facilitate the writing of knowledge and to reuse it in other tools.

On the other hand, the $\mathrm{CP}$ approach allows designers to avoid backtracks in the design process, since criteria are used in the solving process to compute only satisfactory architectures. Thus, designers can choose among several architectures and have a better overview of product possibilities.

\section{References}

Benhamou, F., Goualard, F., Granvilliers, L., Puget, J.-F. (1999). Revising Hull and Box Consistency. In International Conference on Logic Programming, pages 230244. The MIT Press.

Bliek, C., Neveu, B., Trombettoni, G. (1998). Using Graph Decomposition for Solving Continuous CSPs. In CP'98, Pisa, Italy.

Fischer, X., Sebastian, P., Nadeau, J.-P., Zimmer, L. (2004). Constraint based Approach Combined with Metamodeling Techniques to Support Embodiment Design. In $S C I$ '04, Orlando, USA.

Gelle, E., Faltings, B. (2003). Solving Mixed and Conditional Constraint Satisfaction Problems. Constraints, 8:107-141.

Kays, W.M., London, A.L. (1984). Compact Heat Exchangers. Mc Graw-hill book company.

Lhomme, O. (1993). Consistency Techniques for Numeric CSPs. In IJCAI'93, Chambéry, France.

Moore, R. (1966). Interval Analysis. Prentice-Hall.

Neveu, B., Chabert, G., Trombettoni, G. (2006). When Interval Analysis Helps Interblock Backtracking. In $C P^{\prime} 06$, Nantes, France.

O'Sullivan, B. (2001). Constraint-Aided Conceptual Design. Professional Engineering Publishing.

Pahl, G., Beitz, W. (1996). Engineering Design: A Systematic Approach. Springer.

Reddy, S.Y., Fertig K.W., Smith D.E. (1996). Constraint Management Methodology for Conceptual Design Tradeoff Studies. Design Theory and Methodology Conference. Irvine, CA.

Refalo, P. (1999). Tight Cooperation and Its Application in Piecewise Linear Optimization. CP'99, 375-389.

Scaravetti, D., Nadeau, J.P., Sébastian, P., Pailhès, J. (2004). Aided Decision-Making for an Embodiment Design Problem. International IDMME. Bath, UK.

Sébastian, P., Chenouard, R., Nadeau, J.-P., Fischer, X. (2006). The Embodiment Design Constraint Satisfaction Problem of the BOOTSTRAP facing interval analysis and Genetic Algorithm based decision support tools, Proceedings of Virtual Concept 2006, Mexico.

Szykman, S., Racz, J.W., Sriram, R.D. (1999). The Representation of Function in Computer-based Design. Proceedings of the 1999 ASME Design Engineering Technical Conferences (11th International Conference on Design Theory and Methodology). DETC99/DTM-8742.

Van-Hentenryck, P., Mc Allester, D., Kapur, D. (1997). Solving Polynomial Systems Using Branch and Prune Approach, SIAM Journal on Numerical Analysis, vol. $34(2)$, p. $797-827$. 
Vu, X.-H. (2005). Rigorous Solution Techniques for Numerical Constraint Satisfaction Problems. PhD thesis, Swiss Federal Institute of Technology in Lausanne (EPFL). Yannou, B., Simpson, T. W., Barton, R. R.(2003). Towards a Conceptual Design Explorer using Metamodeling Approaches and Constraint Programming. In $A S M E$ DETC'03, Chicago, USA.

Zimmer, L. Zablit, P. (2001). Global Aircraft Predesign based on Constraint Propagation and Interval Analysis. In $C E A S-M A D O$ '01, Koln, Germany. 